\title{
Inserción del Diu por Personal Paramédico Estudio 507
}

\author{
Corporación Centro Regional de Población \\ Area Biomédica \\ Programa Regional de Investigaciones en Fecundidad \\ Dres.: Germán Uriza Gutiérrez, Germán Riaño Gamboa \\ y Lic. Elsy de Barriga
}

\section{INTRODUCCION}

Hace 9 años se realizó en Colombia la primera reunión de coordinadores de programas de planificación familiar en las escuelas de medicina, a la cual asistieron delegados de casi todas las asociaciones afiliadas. En esa reunión se nombró una comisión de especialistas en el campo de la planificación familiar y de la pedagogía médica, que después de varias reuniones de trabajo presentaron en la segunda reunión de coordinadores realizado en mayo de 1974, el anteproyecto sobre el curriculum básico en planificación familiar a nivel de pre-grado en las facultades y escuelas de ciencias de la sálud.

De la segunda reunión nació el plan básico para la enseñanza de la planificación familiar en las facultades (escuelas) de medicina, publicado por la Federación Panamericana de Asociaciones de Facultades (escuelas) de medicina, programa de docencia e investigación en población, en junio de 1974 (4).

Por diversos motivos que no son del caso analizar en este trabajo, el plan básicc para la enseñanza de la planificación familiar en las facultades de medicina de nuestro país, no se ha cumplido; por otra parte, el personal médico que labora en nuestros hospitales, y que no recibe la suficiente preparación en mitodología anticonceptiva en las facultades de medicina, aduce siempre la excesiva carga asistencial y el aumento de las urgencias médico-quirúrgicas como razones que le impiden cubrir adecuadamente la solicitud de servicio en el área de la anticoncepción.

A pesar de la reducción en la tasa bruta de natalidad, la población femenina de 15 a 49 años y la población femenina en unión sigue aumentando (población femenina en riesgo de concebir). Comparando las aceptantes requeridas y las usuarias requeridas, con las usuarias $v$ aceptantes de los programas de Minsalud y Profamilia, se aprecia que la necesidad insatisfecha sobre ningún método anticonceptivo es en $1980 \mathrm{del}$ $29 \%$ o sea 825.000 mujeres, y sobre ningún método que brinde seguridad asciende al $35 \%$ o sea 996.000 mujeres (10). 
Las cifras anteriores demuestran la necesidad de ampliar coberturas en el área de la planificación familiar, pero esta ampliación exige aumento del personal preparado para suministrar el servicio, creando la necesidad de delegar funciones en el personal paramédico. Las enfermeras desarrollan un papel importante para que la gente logre ejercitar su derecho a limitar la prole. Su labor como guardianes de la salud y como instructoras para asegurar la salud, las ponen en contacto diario con pacientes que se encuentran en condiciones tales que aceptan de buen grado sus consejos. Para llevar a cabo su misión, su entrenamiento habrá de abarcar varios y amplios aspectos de planificación familiar, en la teoría y en la práctica (9).

La Corporación Centro Regional de Población, a través del Programa Regional de Investigaciones en Fecundidad, ante la necesidad de actualizar a las enfermeras que ya se encontraban laborando en distintas instituciones del país en programas materno-infantiles, realizó siete cursos de actualización en maternoinfantil y planificación familiar para enfermeras licenciadas, que se realizaron según el siguiente calendario: Bogotá del 21 al 13 de septiembre de 1981; Medellín del 11 al 13 de septiembre de 1980; Cartagena del 27 al 29 de noviembre de 1980; Cali del 5 al 7 de febrero de 1981; MedeIlín del 11 al 13 de junio de 1981; Cali del 26 al 28 de noviembre de 1981; Cali del 25 al 27 de marzo de 1982. En estos cursos se actualizaron un total de 256 enfermeras licenciadas y la evaluación de las mismas demostró que fueron un rotundo éxito, comprobando al mismo tiempo que en varias instituciones de nuestro país las enfermeras brindan servicio a la comunidad en metodología anticonceptiva.

La demanda de servicios de planificación familiar por parte de las usuarias, especialmente en lo relacionado al dispositivo intrauterino, ha hecho que para este tipo de programas sean delegadas funciones al personal paramédico con el debido adiestramiento $y$ supervisión, en áreas que hasta ahora estaban reservadas al médico.

La experiencia en muchas partes del mundo con proyectos piloto en aplicación del DIU por personal paramédico ha sido plenamente satisfactoria. Las ratas de expulsión, embarazo, extracción por dolor o hemorragia y las complicaciones como infección y perforación, son similares comparando los DIUs aplicados por personal no médico, con los DIUs aplicados por personal médico (1) (2) (3) (4) (5) (6) (7) (8) (11) (12) (13) (14) (15) (16) (17) (18) (19) (21). En nuestro medio, Profamilia ha realizado proyectos similares $y$ han demostrado la bondad de los mismos que se traducen en mayor cubrimiento de la demanda con disminución de la relación costobeneficio.

Por lo anteriormente expuesto, se diseñó un proyecto piloto de aplicación de DIU por personal paramédico, en un hospital general donde y'a estaba establecida una consulta de anticoncepción.

\section{OBJETIVOS}

\section{A. Obje tivo General}

Conocer la aceptación de un programa de aplicación del DIU por personal paramédico en nuestro medio y cuantificar las complicaciones, a fin de compararlas con lo encontrado en otras partes del mundo.

\section{B. Objetivos específicos}

1. Conocer la aceptación que en el Hospital General de Medellín pueda tener este tipo de programa en manos del personal paramédico.

2. Desarrollar en este tipo de personal la capacidad de investigación mediante 
la utilización de formularios precodificados.

3. Conocer la aceptabilidad en término de complicaciones y seguimientos, de el DIU tipo lippes aplicado por personal paramédico.

\section{METODOLOGIA}

Adiestramiento de $\mathbf{4}$ auxiliares de enfermería de la consulta de planificación familiar del Hospital General de Medellín en las técnicas de aplicación del DIU bajo la supervisión de una jefe de enfermería y de la doctora Libia Restrepo de Ramírez, Gineco-obstetra y Jefe del depto. de Ginecología y Obstetricia del Hospital General de Medell ín.

La inserción de los dispositivos se hará con la aceptación voluntaria de las pacientes y con la información previa que de rutina se da en los servicios de planificación familiar en relación a este método.
La fecha de iniciación del proyecto será el 15 de julio de 1980, y el último seguimiento será realizado a los 7 meses, febrero 1981, fecha en que debe ser terminado el estudio.

\section{RESULTADOS}

\section{Admisión y seguimientos}

De los 300 DIUs aplicados, se controlaron al mes $266(88.7 \%)$, no cumpliendo el control $34(11.3 \%)$. A los 3 meses con control $188(62.7 \%)$ y sin control $112(37.3 \%)$. A los 6 meses con control $146(48.7 \%)$ y $\sin$ control $154(51.3 \%)$. (Gráfico 1).

\section{Distribución por edad}

Se trata de una población joven, en su mayoría entre los 20 y los 29 años, con un promedio de 24.9 años. (Tabla No. 1). El grupo de menores de 18 años (adolescentes) fue de 32 pacientes, que constituyen el $10.7 \%$ del total.

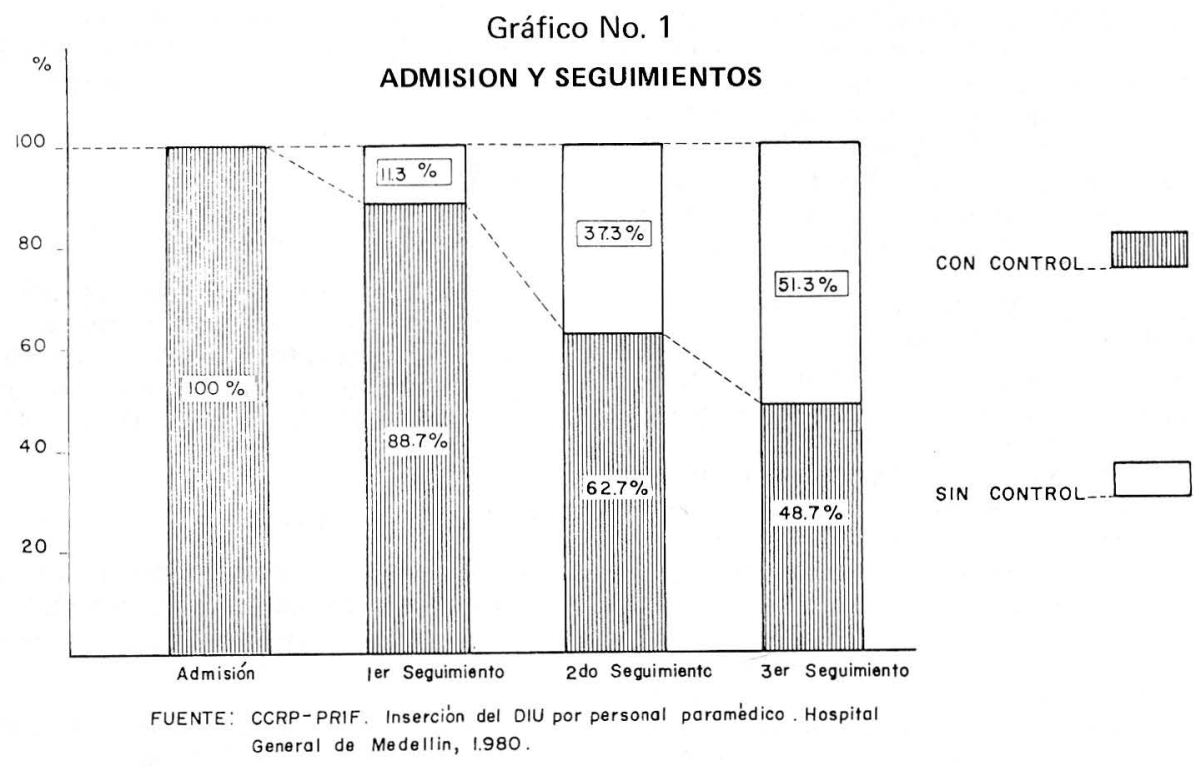


Tabla No. 1

PACIENTES SEGUN DISTRIBUCION POR EDAD

\begin{tabular}{ccc}
\hline $\begin{array}{c}\text { Edad } \\
\text { (años cumplidos) }\end{array}$ & $\begin{array}{c}\text { Número } \\
\text { Pacientes }\end{array}$ & $\%$ \\
\hline $15-19$ & 50 & 16.7 \\
$20-24$ & 111 & 37.1 \\
$25-29$ & 74 & 24.8 \\
$30-34$ & 47 & 15.7 \\
$35-39$ & 13 & 4.4 \\
$40-44$ & 4 & 1.3 \\
\hline TOTAL & 299 & 100.0 \\
\hline
\end{tabular}

No respuesta $=1$

FUENTE: CCRP-PRIF. Inserción del DIU por Personal Paramé dico. Hospital General de Medellín, 1980.

Tabla No. 2

PACIENTES SEGUN NUMERO DE NACIDOS VIVOS

\begin{tabular}{cccc}
\hline $\begin{array}{c}\text { Nacidos } \\
\text { Vivos }\end{array}$ & $\begin{array}{c}\text { Número } \\
\text { Pacientes }\end{array}$ & $\%$ & $\begin{array}{r}\text { Número Total de } \\
\text { Nacidos Vivos }\end{array}$ \\
\hline Ninguno & 2 & 0.7 & 0 \\
1 & 114 & 38.0 & 114 \\
2 & 96 & 32.0 & 192 \\
3 & 50 & 16.6 & 150 \\
4 & 20 & 6.6 & 80 \\
5 & 8 & 2.7 & 40 \\
6 & 3 & 1.0 & 35 \\
7 & 5 & 1.7 & 16 \\
8 & 2 & 0.7 & 645 \\
\hline TOTAL & & & \\
\hline
\end{tabular}

FUENTE: CCRP-PRIF. Inserción del DIU por Personal Paramé dico. Hospital General de Medellin, 1980. 


\section{Número de nacidos vivos}

La mayoría de las pacientes tenían uno y dos hijos. Con un hijo $114(38 \%)$ $y$ con dos hijos $96(32 \%)$. Las grandes multíparas ( 5 o más hijos) fueron solo $18(6.1 \%)$. Las dos pacientes que aparecen sin hijos tenían antecedente de mortinato. El total de nacidos vivos para las 300 pacientes fue de 645 , o sea un promedio de 2.15 hijos por paciente. (Tabla No. 2).

\section{Número de embarazos a término}

Sólo se encuentran dos pacientes con antecedente de parto prematuro que constituyen el $0.7 \%$ del total (Tabla No. $3)$.

\section{Número de abortos inducidos y espontáneos}

La mayoría de las pacientes, 218 $(72.7 \%)$, no tenían antécedentes de aborto. Con un aborto, $53(17.7 \%)$, y con dos o más abortos, $29(9.6 \%)$. El número total de pacientes con antecedentes de aborto fue de $82(27.3 \%)$. (Tabla No. 4).

\section{Anticonceptivo usado previamente a la inserción del DIU}

La mayoría $185(61.7 \%)$, no usaban ningún método anticonceptivo. De las 115 pacientes que utilizaban algún ımétodo anticonceptivo $(38.3 \%)$, usaban un método de alta efectividad 90 pacientes $(30 \%)$ y de baja efectividad 25 $(8.3 \%)$. (Tabla No. 5).

7. Resultado del último embarazo previo a la inserción del DIU

No se aplicó DIU en ninguna nulípara $y$ las dos pacientes que se encontraron sin hijos tenían antecedente de mortinato. La gran mayoría de las pacientes, $268(89.3 \%)$, tenían como resultado del último embarazo un hijo vivo. (Tabla No. 6).

Tabla No. 3

PACIENTES SEGUN NUMERO DE EMBARAZOS A TERMINO

\begin{tabular}{crrr}
\hline $\begin{array}{c}\text { Embarazo a } \\
\text { Término }\end{array}$ & $\begin{array}{c}\text { Número } \\
\text { Pacientes }\end{array}$ & $\%$ & $\begin{array}{c}\text { Total Número } \\
\text { de Embarazos }\end{array}$ \\
\hline Ninguno & 2 & 0.7 & 0 \\
1 & 117 & 39.0 & 117 \\
2 & 91 & 30.3 & 182 \\
3 & 51 & 17.0 & 153 \\
4 & 20 & 6.6 & 80 \\
5 & 9 & 3.0 & 45 \\
6 & 3 & 1.0 & 18 \\
7 & 5 & 1.7 & 35 \\
8 & 2 & 0.7 & 16 \\
\hline TOTAL & 300 & 100.0 & 646 \\
\hline
\end{tabular}

FUENTE: CCRP-PRIF. Inserción del DIU por Personal Paramédico. Hospital General de Medellín, 1980. 
Tabla No. 4

PACIENTES SEGUN NUMERO DE ABORTOS INDUCIDOS Y ESPONTANEOS

\begin{tabular}{cccc}
\hline Abortos & $\begin{array}{c}\text { Número } \\
\text { Pacientes }\end{array}$ & $\%$ & $\begin{array}{c}\text { Número Total } \\
\text { de Abortos }\end{array}$ \\
\hline Ninguno & 218 & 72.7 & 0 \\
1 & 53 & 17.7 & 53 \\
2 & 19 & 6.3 & 38 \\
3 & 7 & 2.3 & 21 \\
4 & 1 & 0.3 & 4 \\
5 & 2 & 0.7 & 10 \\
\hline \multirow{2}{*}{ TOTAL } & & & 126 \\
\hline
\end{tabular}

FUENTE: CCRP-PRIF. Inserción del DIU por Personal Paramédico. Hospital General de Medellin, 1980.

Tabla No. 5

PACIENTES SEGUN ANTICONCEPTIVO USADO PREVIAMENTE A LA INSERCION DEL DIU

\begin{tabular}{lcr}
\hline $\begin{array}{l}\text { Método Anticonceptivo } \\
\text { Usado }\end{array}$ & $\begin{array}{c}\text { Número } \\
\text { Pacientes }\end{array}$ & $\%$ \\
\hline Ninguno & 185 & 61.7 \\
DIU & 24 & 8.0 \\
Orales & 63 & 21.0 \\
Inyectables & 3 & 1.0 \\
Condón & 6 & 2.0 \\
Espuma & 1 & 0.3 \\
Otros métodos de barrera & 3 & 1.0 \\
Otro & 15 & 5.0 \\
\hline TOTAL & 300 & 100.0 \\
\hline
\end{tabular}

FUENTE: CCRP-PRIF. Inserción del D.IU por Personal Paramédi co. Hospital General de Medellín, 1980. 
Tabla No. 6

PACIENTE SEGUN EL RESULTADO DEL ULTIMO EMBARAZO

PREVIO A LA INSERCION DEL DIU

\begin{tabular}{lrr}
\hline \multicolumn{1}{c}{ Resultado } & Número & $\%$ \\
Ultimo Embarazo & Pacientes & $\%$ \\
\hline Nacido vivo & 268 & 89.3 \\
Mortinato & 2 & 0.7 \\
Aborto espontáneo & 24 & 8.0 \\
Aborto séptico & 5 & 1.7 \\
Embarazo ectópico & 1 & 0.3 \\
& & \\
\hline TOTAL & 300 & 100.0 \\
\hline
\end{tabular}

FUENTE: CCRP-PRIF. Inserción del DIU por Personal Paramédi co. Hospital General de Medellín, 1980.

\section{Pacientes lactando actualmente}

La mayoría de las pacientes se encontraban lactando, $184(61.3 \%)$. No estaban lactando $116(38.7 \%)$.

9. Principal queja menstrual previa a la inserción del DIU

La gran mayoría $282(94 \%)$ no tenía ninguna alteración menstrual referente a sangrado anormal o dismenorrea. Con dismenorrea previa $12\left(4^{\circ} \%\right)$ y con sangrado intermenstrual $3(1 \%)$. Dolor pélvico indicativo de ovulación sólo 3 pacientes $(1 \%)$. (Tabla No. 7).

10. Antecedente de inflamación pélvica

La gran mayoría 295 (98.3\%), no tenían ningún antecedente de este trastorno. Cuatro pacientes un solo episodio $(1.3 \%)$ y una paciente con varios episodios $(0.3 \%)$.

\section{Antecedente de enfermedades venéreas}

La gran mayoría $294(98 \%)$, no tenían antecedente venéreo. Sólo 6 pa- cientes anotaban dicho antecedente $(2 \%)$.

12. Tiempo desde la terminación del último embarazo y la inserción del DIU

No se aplicó DIU a ninguna paciente nulípara. En sólo 2 pacientes se aplicó el DIU en el puerperio inmediato $(0.7 \%)$. Se aplicó entre 36 horas y 6 semanas de terminado el embarazo a 91 pacientes $(30.3 \%)$, distribuidas en 10 pacientes de aborto y 81 pacientes de parto. Se aplicó después de 6 o más semanas de terminado el último embarazo a 207 pacientes $(69 \%)$.

\section{Tamaño del DIU}

La distribución entre lippes $\mathrm{C}$ y lippes D fue similar. Con lippes C, 177 pacientes $(59 \%)$ y con lippes D, 123 pacientes $(41 \%)$.

14. Principal problema relacionado con la inserción

No existió ningún problema en casi la totalidad de las pacientes $295(99 \%)$ y 
ninguna inserción fallida; vómito en 2 pacientes $(0.7 \%)$ y mareo en una paciente $(0.3 \%)$.

\section{Dolor pélvico durante la inserción}

La mitad de las pacientes 156 $(52.3 \%)$, no presentó dolor a la inserción del DIU. Dolor leve 88 pacientes $(29.5 \%)$; moderado $49(16.4 \%)$ y severo en sólo 5 pacientes $(1.7 \%)$. (Gráfico No. 2).

\section{Tratamiento de complicaciones o quejas}

La gran mayoría de las pacientes 290 $(97.3 \%)$, no requirieron de ningún tratamiento. Siete pacientes $(2.3 \%)$ requirieron observación y una $(0.4 \%)$, tratamiento médico quirúrgico.
17. Conocimiento previo de otros métodos anticonceptivos

La mayoría de las pacientes 229 $(77.6 \%)$ conocían otros métodos anticonceptivos diferentes del DIU. Sesenta y seis pacientes $(22.4 \%)$, sólo conocían como anticonceptivo el DIU.

18. Proporción de compañeros que aceptan la anticoncepción

En la gran mayoría de las pacientes, $268(91.5 \%)$, los esposos o compañeros están de acuerdo con que su pareja planifique. En 25 pacientes $(8.5 \%)$ los esposos o compañeros se oponen a la anticoncepción.

19. Razón por la cual solicita anticoncepción

La gran mayoría solicita el servicio porque desea espaciar los hijos o no

Tabla No. 7

PACIENTES SEGUN LA PRINCIPAL QUEJA MENSTRUAL PREVIA A LA INSERCION DEL DIU

\begin{tabular}{lcc}
\hline Queja & $\begin{array}{c}\text { Número } \\
\text { Pacientes }\end{array}$ & $\%$ \\
\hline $\begin{array}{l}\text { Ninguna } \\
\begin{array}{l}\text { Manchas/goteo } \\
\text { intermenstrual }\end{array}\end{array}$ & 282 & 94.0 \\
$\begin{array}{l}\text { Sangrado inter- } \\
\text { menstrual }\end{array}$ & 2 & 0.7 \\
$\begin{array}{l}\text { Dismenorrea } \\
\text { Dolor pélvico } \\
\text { intermenstrual }\end{array}$ & 1 & 0.3 \\
\hline TOTAL & 3 & 4.0 \\
\hline
\end{tabular}

FUENTE: CCRP-PRIF. Inserción del DIU por Personal Paramédi co. Hospital General de Medellin, 1980. 
Gráfico No. 2

\section{DOLOR PELVICO DURANTE LA INSERCION}

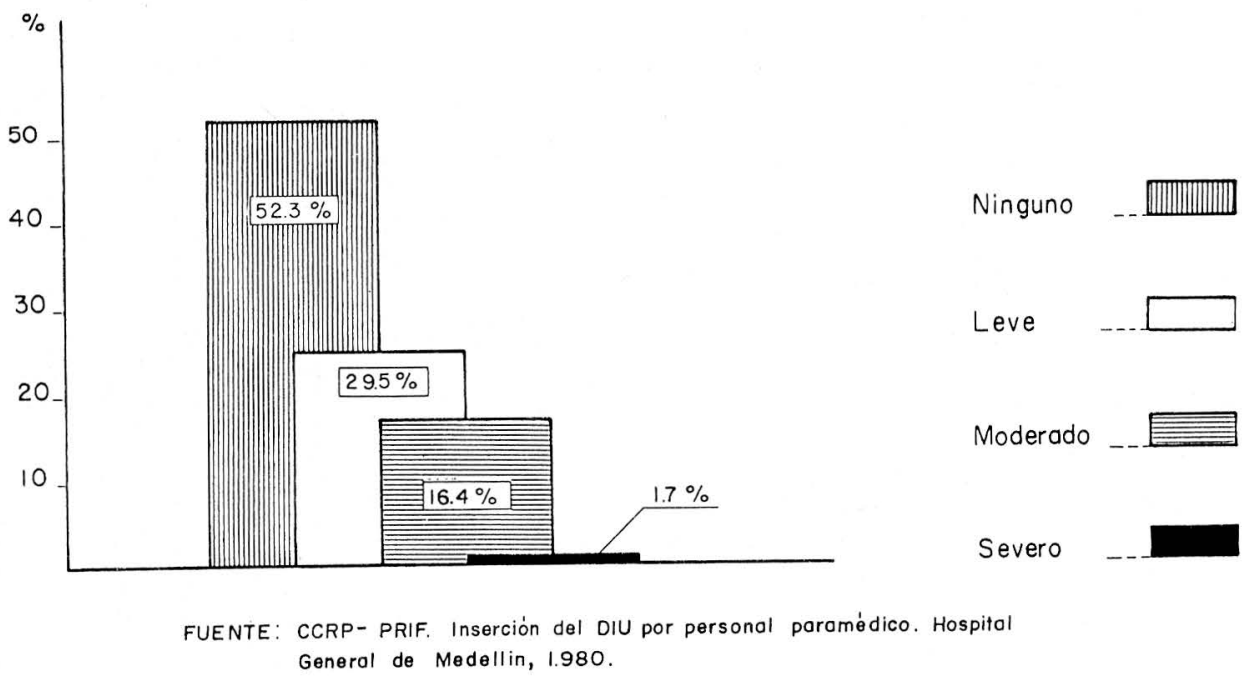

tenerlos más $(80.6 \%)$; distribuidas en 1-6 pacientes que no quieren tener más hijos $(41.9 \%)$ y 112 que desean espaciarlos $(38.7 \%)$. La situación económica sólo la aducen como razón 38 pacientes $(13.1 \%)$ y otras causas sólo 18 pacientes $(6.3 \%)$. (Tabla No. 8).

20. Resultados en el primer seguimiento (al mes de aplicado el DIU)

\subsection{Asistencia al primer control}

Cumplieron este seguimiento 266 pacientes $(88.7 \%$ ). (Gráfico 1$)$. No ocurrió ninguna muerte ni tampoco ningún embarazo.

\subsection{Interrupción del método}

Se interrumpió el método en 19 pacientes $(7.1 \%)$ que corresponden a 14 expulsiones $(5.3 \%)$ y 5 retiros $(1.9 \%)$, distribuidas en la siguiente forma: Por dolor 2 pacientes $\left(0.7^{\circ} \%\right)$, por razones personales 2 pacientes $(0.7 \%)$, embarazo planeado una paciente $(0.4 \%)$. (Tabla No. 9).

\subsection{Sangrado o dolor}

Sangrado anormal y dolor lo presentaron 85 pacientes $(32 \%)$ discriminados en la siguiente forma: sangrado anormal 51 pacientes $(19.2 \%)$ que corresponden a 24 manchas intermenstruales $(9 \%)$, 21 sangrados intermenstruales $(7.9 \%)$ y 6 hemorragias (2.3), dolor en 34 pacientes $(12.8 \%)$; que corresponden a 24 dismenorreas $(9 \%)$ y 10 dolor pélvico intermenstrual $(3.8 \%)$. Sin sangrado ni dolor 181 pacientes $(68 \%)$ ). (Tabla No. 10).

\subsection{Infección pélvica y enfermedad venérea}

Ninguna paciente presentó enfermedad inflamatoria pélvica. En sólo 2 pa- 
Tabla No. 8

PACIENTES SEGUN LA RAZON POR LA CUAL SOLICITAN ANTICONCEPCION

\begin{tabular}{lcc}
\hline Razón Principal & $\begin{array}{c}\text { Número } \\
\text { Pacientes }\end{array}$ & $\%$ \\
\hline No desea más hijos & 121 & 41.9 \\
Espaciar hijos & 112 & 38.7 \\
Situación económica & 38 & 13.1 \\
Por la edad & 2 & 0.7 \\
Por lactancia & 8 & 2.8 \\
Problemas personales & 4 & 1.4 \\
Enfermedad & 4 & 1.4 \\
\hline TOTAL. & 289 & 100.0 \\
\hline
\end{tabular}

No respuesta $=11$

FUENTE: CCRP-PRIF. Inserción del DIU por Personal Paramédico. Hospital General de Medellín, 1980.

Tabla No. 9

PACIENTES DE PRIMER CONTROL SEGUN INTERRUPCION DEL METODO

\begin{tabular}{lcc}
\hline Retiro del DIU por: & $\begin{array}{c}\text { Número } \\
\text { Pacientes }\end{array}$ & $\%$ \\
\hline No retirado & 247 & 92.9 \\
Dolor & 2 & 0.7 \\
Embarazo planificado & 1 & 0.4 \\
Otras razones personales & 2 & 0.7 \\
Expulsión & 14 & 5.3 \\
& & \\
\hline TOTAL & 266 & 100.0
\end{tabular}

FUENTE: CCRP-PRIF. Inserción del DIU por Personal Paramé dico. Hospital General de Medellín, 1980. 
cientes se comprobó enfermedad venérea $(0.8 \%)$ y ésta fue trichomoniasis.

20.5 Otros problemas relacionados con el DIU

En relación a otros problemas referentes al DIU, el único fue el flujo vaginal. Sin especificar el gérmen causal, que lo presentaron 7 pacientes $(2.6 \%)$.

20.6 Tratamiento de la complicación o queja

El tratamiento de las complicaciones o quejas fue sólo la observación con una nueva cita de control, no se requirió ni hospitalización ni cirugía en ninguna paciente.

20.7 Satisfacción de las pacientes con el método

De las 266 pacientes que asistieron al primer control, se encontraron satisfechas con el DIU $242(90.9 \%)$ y no satisfechas $24(9.1 \%)$ que corresponden a 14 expulsiones $(5.3 \%), 5$ retiros $(1.9 \%)$ y 5 no respuestas $(1.9 \%)$.
20.8 Facilidad para cumplir los controles

De las pacientes que asistieron al control, tienen facilidad para cumplir esta cita 198 pacientes $(74.4 \%)$ y encuentran dificultad $44(16.5 \%)$.

\subsection{Dificultad para cumplir los} controles

De las pacientes con dificultad para cumplir la cita, se encuentra relacionada a condiciones socio-económicas, en 32 pacientes $(72.7 \%$ ) que corresponden a 15 que no tienen con quién dejar los hijos $(34.1 \%)$, problemas económicos $10(22.7 \%), 6$ que trabajan $(13.6 \%)$ y una que no tiene quién cuide la casa $(2.3 \%)$. (Tabla No. 11$)$.

21. Resultados en el segundo seguimiento la los 3 meses de aplicado el DIU)

\subsection{Asistencia al segundo control}

Al segundo control asistieron 188 pacientes $(62.7 \%$ ). (Gráfico No. 1). No

Tabla No. 10

PACIENTES DE PRIMER CONTROL SEGUN SANGRADO Y DOLOR

\begin{tabular}{lcr}
\hline Sangrado y Dolor & $\begin{array}{c}\text { Número } \\
\text { Pacientes }\end{array}$ & $\%$ \\
\hline Ninguno & 181 & 68.0 \\
Manchas intermenstruales & 24 & 9.0 \\
Sangrados intermenstruales & 21 & 7.9 \\
Hemorragias & 6 & 2.3 \\
Dismenorrea & 24 & 9.0 \\
Dolor pélvico & 10 & 3.8 \\
& & \\
\hline TOTAL & 266 & 100.0 \\
\hline
\end{tabular}

FUENTE: CCRP-PRIF. Inserción del DIU por Personal Paramé dico. Hospital General de Medellin, 1980. 
Tabla No. 11

PACIENTES DE PRIMER CONTROL SEGUN DIFICULTAD PARA CUMPLIR LOS CONTROLES

\begin{tabular}{|c|c|c|}
\hline Dificultad & $\begin{array}{c}\text { Número } \\
\text { Pacientes }\end{array}$ & $\%$ \\
\hline $\begin{array}{l}\text { No tiene quien cuide hijos } \\
\text { Problemas económicos } \\
\text { Trabajo } \\
\text { Sufre de várices } \\
\text { Vive lejos } \\
\text { Viaja } \\
\text { No tiene tiempo } \\
\text { Esposo celoso } \\
\text { No tiene quien cuide casa } \\
\text { Enfermedad }\end{array}$ & $\begin{array}{r}15 \\
10 \\
6 \\
1 \\
4 \\
2 \\
3 \\
1 \\
1 \\
1\end{array}$ & $\begin{array}{r}34.1 \\
22.7 \\
13.6 \\
2.3 \\
9.1 \\
4.5 \\
6.8 \\
2.3 \\
2.3 \\
2.3\end{array}$ \\
\hline TOTAL & 44 & 100.0 \\
\hline $\begin{array}{l}\text { NOTA: Ninguna dificultad } \\
\text { Expulsiones y retiros } \\
\text { No respuesta } \\
\text { Total }\end{array}$ & $\begin{array}{r}198 \text { pacientes } \\
19 \text { pacientes } \\
5 \text { pacientes } \\
222\end{array}$ & \\
\hline
\end{tabular}

FUENTE: CCRP-PRIF. Inserción del DIU por Personal Paramé dico. Hospital General de Medellin, 1980.

ocurrió ninguna muerte, ni tampoco ningún embarazo.

\subsection{Interrupción del método}

Se interrumpió el método en 9 pacientes $(4.7 \%)$ que corresponden a: 4 expulsiones $(2.1 \%)$ y 5 retiros $(2.6 \%)$ distribuidos en la siguiente forma: 2 desplazamientos al canal cervical $(1.1 \%)$, una por dolor $(0.5 \%)$, una por embarazo planeado $(0.5 \%)$ y una por razones personales $(0.5 \%)$. (Tabla No. 12).

\subsection{Sangrado o dolor}

Sangrado anormal o dolor lo presentaron 57 pacientes $(30.3 \%)$, distribui- das en la siguiente forma: sangrado anormal 31 pacientes $(16.5 \%)$ que corresponden a 13 manchas intermenstruales $(6.9 \%), 10$ sangrados intermenstruales $(5.3 \%) 6$ hemorragias $(3.2 \%)$ y 2 hipermenorreas $(1.1 \%)$. Dolor lo presentaron 26 pacientes $(13.8 \%)$ que corresponde a 17 dismenorreas $(9 \%)$, dolor pélvico intermenstrual 8 pacientes $(4.3 \%)$ y una paciente con dolor en la cintura $(0.5 \%)$. (Tabla No. 13).

\subsection{Infección pélvica y enfermedad venérea}

Enfermedad inflamatoria pélvica la presentaron 2 pacientes $(1.1 \%)$, ambas estado II (útero y anexos). Enfermedad 
Tabla No. 12

PACIENTES DE SEGUNDO CONTROL SEGUN

INTERRUPCION DEL METODO

\begin{tabular}{lcc}
\hline Retiro DIU por: & $\begin{array}{c}\text { Número } \\
\text { Pacientes }\end{array}$ & $\%$ \\
\hline No retirado & 179 & 95.2 \\
Desplazamiento & 2 & 1.1 \\
Dolor & 1 & 0.5 \\
Embarazo planificado & 1 & 0.5 \\
Razón personal (murió esposo) & 1 & 0.5 \\
Expulsión & 4 & 2.1 \\
& & \\
\hline TOTAL & 188 & 100.0 \\
\hline
\end{tabular}

FUENTE: CCRP-PRIF. Inserción del DIU por Personal Paramédico. Hospital General de Medellín, 1980.

Tabla No. 13

PACIENTES DE SEGUNDO CONTROL SEGUN SANGRADO Y DOLOR

\begin{tabular}{lcc}
\hline Sangrado y Dolor & $\begin{array}{c}\text { Número } \\
\text { Pacientes }\end{array}$ & $\%$ \\
\hline Ninguno & 131 & 69.7 \\
Manchas intermenstruales & 13 & 6.9 \\
Sangrado intermenstrual & 10 & 5.3 \\
Hemorragias & 6 & 3.2 \\
Dismenorrea & 17 & 9.0 \\
Dolor pélvico intermenstrual & 8 & 4.3 \\
Hipermenorreas & 2 & 1.1 \\
Dolor de cintura & 1 & 0.5 \\
& & \\
\hline TOTAL & 188 & 100.0 \\
\hline
\end{tabular}

FUENTE: CCRP-PRIF. Inserción del DIU por Personal Paramédico. Hospital General de Medellin, 1980. 
venérea 2 pacientes $(1.1 \%)$, ambas trichomoniasis.

\subsection{Otros problemas relacionados con el DIU}

Estos fueron: amenorrea en una sola paciente $(0.5 \%)$; leucorrea en una sola paciente $(0.5 \%)$ y sin especificar en una paciente $(0.5 \%)$.

\subsection{Tratamiento de la complicación o queja}

Se trataron 13 complicaciones $(6.9 \%)$, 8 pacientes $(4.2 \%)$, sólo requirieron observación con una nueva cita de control. Recibieron tratamiento 5 pacientes $\left(2.7^{\circ} \%\right)$, con ciclos hormonales 2 pacien- tes, sin especificar 2 pacientes y con óvulos una paciente.

21.7 Satisfacción de las pacientes con el método

De las 188 pacientes que asistieron al segundo control, se encontraron satisfechas con el método $174(92.5 \%)$; las otras 14 pacientes corresponden a 9 expulsiones $(4.8 \%)$ y 5 no respuestas $(2.7 \%)$.

21.8 Facilidad para cumplir los controles

De las 188 pacientes que asistieron al segundo control tienen facilidad para

Tabla No. 14

PACIENTES DE SEGUNDO CONTROL SEGUN DIFICULTAD PARA CUMPLIRLO

\begin{tabular}{lcc}
\hline Dificultad & $\begin{array}{c}\text { Número } \\
\text { Pacientes }\end{array}$ & $\%$ \\
\hline
\end{tabular}

$\begin{array}{llr}\text { No tiene quien cuide hijos } & 7 & 23.3 \\ \text { Económico } & 5 & 16.7 \\ \text { Trabajando } & 5 & 16.7 \\ \text { Sufre de várices } & 1 & 3.3 \\ \text { Vive muy lejos } & 4 & 13.3 \\ \text { Viaja } & 2 & 6.7 \\ \text { No tiene tiempo } & 3 & 10.0 \\ \text { Esposo celoso } & 1 & 3.3 \\ \text { No tiene quien cuide la casa } & 1 & 3.3 \\ \text { Asma bronquial } & 1 & 3.3\end{array}$

$\begin{array}{lll}\text { TOTAL } & 30 & 100.0\end{array}$

\begin{tabular}{|c|c|c|c|}
\hline TA : & $\begin{array}{l}\text { Ninguna dificultad } \\
\text { Expulsiones } \\
\text { No respuesta }\end{array}$ & $\begin{array}{r}148 \\
9 \\
1\end{array}$ & $\begin{array}{l}\text { pacientes } \\
\text { pacientes } \\
\text { paciente }\end{array}$ \\
\hline & & 15 & \\
\hline
\end{tabular}

FUENTE: CCRP-PRIF. Inserción del DIU por Personal Paramédico. Hospital General de Medellin, 1980. 
cumplir la cita $144\left(76.6^{\mathrm{U}} \%\right)$ y encuentran dificultad $30(15.9 \%)$.

\subsection{Dificultad para cumplir controles}

De las 30 pacientes que encuentran dificultad para cumplir las citas, éstas están relacionadas con las condiciones socio-económicas de las pacientes en 18 casos $(60 \%)$ que corresponden a 7 que no tienen con quién dejar los hijos $(23.3 \%)$, problemas económicos 5 $(16.7 \%)$, trabajan $5(16.7 \%)$ y no tiene quién cuide la casa, una paciente (3.3). (Tabla No. 14).

22. Resultados del tercer seguimiento (a los 6 meses de aplicado el DIU).

\subsection{Asistencia al tercer control}

Al tercer seguimiento asistieron 146 pacientes $(48.7 \%)$. (Gráfico No. 1). No ocurrió ninguna muerte y se presentó un embarazo $(0.7 \%)$ por DIU expulsado en la vagina.

\subsection{Interrupción del método}

Se interrumpió el método en 23 pacientes $(15.8 \%)$ que corresponden a 7 expulsiones $(4.8 \%)$ y 16 retiros $(11 \%)$, 6 razones personales $(4.1 \%), 3$ desplazamientos al canal cervical $(2.0 \%), 3$ anexitis $(2.0 \%), 2$ hemorragias $(1.4 \%)$ y 2 embarazos planeados $(1.4 \%)$. (Tabla No. 15).

\subsection{Sangrado o dolor}

Sangrado anormal o dolor lo presentaron 53 pacientes $(36.3 \%)$ distribuidas en la siguiente forma: sangrado anormal 23 pacientes $(15.7 \%)$, que corresponden a 14 manchas intermenstruales $(9.6 \%)$, 5 hemorragias $(3.4 \%), 3$ sangrados intermenstruales $(2.1 \%)$ y una hipermenorrea $(0.7 \%)$. Dolor lo presentaron 29 pacientes $(19.9 \%)$ que corresponden a 19 dismenorreas $(13 \%)$ y 10 con dolor pélvico intermenstrual $(6.8 \%)$. En un caso no se especificó el tipo de alteración $(0.7 \%$ ). (Tabla No. 16).

Tabla Nio. 15

\section{PACIENTES DE TERCER CONTROL SEGUN INTERRUPCION DEL METODO}

\begin{tabular}{lcr}
\hline Retiro del DIU & $\begin{array}{c}\text { Número } \\
\text { Pacientes }\end{array}$ & $\%$ \\
\hline No retirado & 123 & 84.2 \\
Desplazamiento & 3 & 2.1 \\
Sangrado & 2 & 1.4 \\
Anexitis & 3 & 2.0 \\
Embarazo planificado & 2 & 1.4 \\
Otras razones personales & 6 & 4.1 \\
Expulsión & 7 & 4.8 \\
& & 146 \\
\hline
\end{tabular}

FUENTE: CCRP-PRIF. Inserción del DIU por Personal Paramé dico. Hospital General de Medellín, 1980. 
Tabla No. 16

PACIENTES DE TERCER CONTROL SEGUN DOLOR Y SANGRADO

\begin{tabular}{lcr}
\hline Sangrado y Dolor & $\begin{array}{c}\text { Número } \\
\text { Pacientes }\end{array}$ & $\%$ \\
\hline Ninguno & 93 & 63.7 \\
Manchas intermenstruales & 14 & 9.6 \\
Sangrado intermenstrual & 3 & 2.1 \\
Hemorragias & 5 & 3.4 \\
Dismenorrea & 19 & 13.0 \\
Dolor pélvico intermenstrual & 10 & 6.8 \\
Hipermenorrea & 1 & 0.7 \\
Sin especificar & 1 & 0.7 \\
& & \\
\hline TOTAL & 146 & 100.0 \\
\hline
\end{tabular}

FUENTE: CCRP-PRIF. Inserción del DIU por Personal Paramé dico. Hospital General de Medellín, 1980.

\subsection{Infección pélvica y entermedad} venérea

Enfermedad inflamatoria pélvica la presentaron 4 pacientes $(2.7 \%)$ que corresponden a EIP estado I (sólo uterina), una paciente $(0.7 \%$ ) y estado II (útero y anexos) 3 pacientes $(2 \%)$.

Enfermedad venérea sólo la presentó una paciente $(0.7 \%)$, que corresponde a sífilis primaria.

\subsection{Otros problemas relacionados con el DIU}

Estos se presentaron en tres pacientes $(2.1 \%)$ que corresponden a: una leucorrea $(0.7 \%)$, una dispareunia $(0.7 \%)$ y sin especificar una $(0.7 \%)$.

\subsection{Tratamiento de la complicación o queja}

Se trataron 9 pacientes $(6.2 \%)$, distribuidas en la siguiente forma: una observación $(0.7 \%), 5$ tratamientos mé- dicos $(3.4 \%)$, que corresponden a 3 tratamientos antibióticos y 2 tratamientos cíclicos hormonales y 3 tratamientos quirúrgicos $(2.1 \%)$, todos legrados uterinos. (Tabla No. 17).

\subsection{Satisfacción de las pacientes con el método}

De las 146 pacientes que cumplieron el tercer seguimiento se encontraron satisfechas $120(82.2 \%)$, las restantes corresponden a 23 expulsiones $(15.7 \%)$ y 3 no respuestas $(2.1 \%)$.

\subsection{Facilidad para cumplir los controles}

De las 146 pacientes que asistieron al tercer seguimiento, tienen facilidad para cumplir la cita $99\left(67.8^{\circ} \%\right)$ y encuentran dificultad 21 pacientes $(21.4 \%$ ).

\subsection{Dificultad para cumplir los controles}

De las 21 pacientes que encuentran dificultad para cumplir la cita, éstas es- 
Tabla No. 17

\section{PACIENTES DE TERCER CONTROL QUE NECESITAN}

TRATAMIENTO DE LA COMPLICACION O QUEJA

\begin{tabular}{lcc}
\hline Tratamiento & $\begin{array}{c}\text { Número } \\
\text { Pacientes }\end{array}$ & $\%$ \\
\hline No hubo complicación o queja & 137 & 93.8 \\
Solo observación & 1 & 0.7 \\
Médico $\left\{\begin{array}{l}\text { Antibióticos } \\
\text { Ciclos hormonales }\end{array}\right.$ & 3 & 2.1 \\
Quirúrgico (curetajes) & 3 & 1.3 \\
\hline TOTAL & 146 & 100.0 \\
\hline
\end{tabular}

FUENTE: CCRP-PRIF. Inserción del DIU por Personal Paramé dico. Hospital General de Medellin, 1980.

Tabla No. 18

PACIENTES DE TERCER CONTROL SEGUN DIFICULTAD PARA CUMPLIRLO

\begin{tabular}{lcr}
\hline Dificultad & $\begin{array}{c}\text { Número } \\
\text { Pacientes }\end{array}$ & $\%$ \\
\hline No tiene quien cuide hijos & 5 & 23.8 \\
Económico & 3 & 14.3 \\
Trabajando & 3 & 14.3 \\
Sufre de várices & 1 & 4.8 \\
Vive muy lejos & 4 & 19.0 \\
Viaja & 1 & 4.8 \\
No tiene tiempo & 3 & 14.3 \\
Asma bronquial & 1 & 4.8 \\
\hline TOTAL & 21 & 100.0 \\
\hline
\end{tabular}

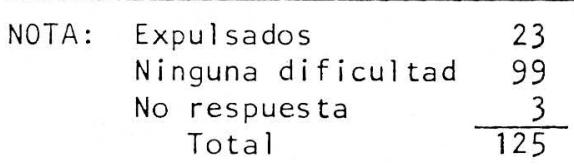

FUENTE: CCRP-PRIF. Inserción del DIU por Personal Paramé dico. Hospital General de Medellin, 1980. 
Tabla No. 19

PACIENTES POR TIEMPO DE APLICACION DEL DIU DESPUES DE PARTO O ABORTO SEGUN CONTROL

\begin{tabular}{|c|c|c|c|c|c|c|c|c|c|c|c|c|c|}
\hline \multirow{3}{*}{\multicolumn{2}{|c|}{$\begin{array}{l}\text { Tiempo desde Embarazo } \\
\text { y la Inserción del DIU }\end{array}$}} & \multicolumn{8}{|c|}{ CONTROL } & \multicolumn{2}{|c|}{ Subtotal } & \multicolumn{2}{|r|}{ Total } \\
\hline & & \multicolumn{2}{|c|}{ NO } & \multirow{2}{*}{$\frac{010}{\pi}$} & \multirow{2}{*}{$\frac{\mid \text { INSITU }}{\%}$} & \multicolumn{2}{|r|}{$\frac{51}{0 \text { Desplaz" }}$} & \multicolumn{2}{|c|}{ Retiro } & \multirow[b]{2}{*}{$\#$} & \multirow[b]{2}{*}{$\%$} & \multirow[b]{2}{*}{$\#$} & \multirow[b]{2}{*}{$1 \%$} \\
\hline & & \# & $\%$ & & & \# & $1 \%$ & $\#$ & $1 \%$ & & & & \\
\hline 2 a 36 horas & $\begin{array}{l}\text { Parto } \\
\text { Aborto }\end{array}$ & $\begin{array}{l}1 \\
-\end{array}$ & 50.0 & 1 & 50.0 & - & - & - & - & $\begin{array}{l}1 \\
-\end{array}$ & 50.0 & 2 & 100.0 \\
\hline \multicolumn{2}{|l|}{ Subtotal } & 1 & 50.0 & 1 & 50.0 & - & - & - & - & 1 & 50.0 & 2 & 0.7 \\
\hline $\begin{array}{l}\text { Más de } 36 \text { horas } \\
\text { y menos de } 6 \\
\text { semanas }\end{array}$ & $\begin{array}{l}\text { Parto } \\
\text { Aborto }\end{array}$ & 13 & $\begin{array}{l}16.0 \\
10.0\end{array}$ & $\begin{array}{r}54 \\
8\end{array}$ & $\begin{array}{l}66.7 \\
80.0\end{array}$ & $\begin{array}{l}9 \\
-\end{array}$ & 11.1 & $\begin{array}{l}5 \\
1\end{array}$ & $\begin{array}{r}6.2 \\
10.0\end{array}$ & $\begin{array}{r}68 \\
9\end{array}$ & $\begin{array}{l}84.0 \\
90.0\end{array}$ & $\begin{array}{l}81 \\
10\end{array}$ & $\begin{array}{l}89.0 \\
11.0\end{array}$ \\
\hline \multicolumn{2}{|l|}{\begin{tabular}{|l} 
Semanas \\
Subtotal
\end{tabular}} & 14 & 15.4 & 62 & 68.1 & 9 & 9.9 & 6 & 6.6 & 77 & 84.6 & 91 & 30.3 \\
\hline $\begin{array}{l}6 \text { semanas : } \\
\text { más }\end{array}$ & $\begin{array}{l}\text { Parto } \\
\text { Aborto }\end{array}$ & $\begin{array}{r}17 \\
2 \\
\end{array}$ & $\begin{array}{r}9.1 \\
10.5 \\
0.9\end{array}$ & $\begin{array}{r}138 \\
14 \\
153\end{array}$ & $\begin{array}{l}73.4 \\
73.7 \\
\end{array}$ & $\begin{array}{r}20 \\
1 \\
1\end{array}$ & $\begin{array}{r}10.6 \\
5.3 \\
\end{array}$ & $\begin{array}{r}13 \\
2 \\
15\end{array}$ & $\begin{array}{r}6.9 \\
10.5 \\
7.3\end{array}$ & $\begin{array}{r}171 \\
17 \\
\end{array}$ & $\begin{array}{l}90.9 \\
89.5 \\
90.8\end{array}$ & $\begin{array}{r}188 \\
19 \\
207\end{array}$ & $\begin{array}{r}90.8 \\
9.2 \\
\end{array}$ \\
\hline Subtotal & & 19 & 9.2 & 152 & 73.4 & 21 & 10.1 & 15 & 7.3 & 188 & 90.8 & 207 & 69.0 \\
\hline TOTAL & & 34 & 11.3 & 215 & 71.7 & 30 & 10.0 & 21 & 7.0 & 266 & 88.7 & 300 & 100.0 \\
\hline
\end{tabular}

* Expulsión o Desplazamiento.

FUENTE: CCRP-PRIF. Inserción del OIU por Personal Paramédico. Hospltal General de Medellín, 1980.

\section{Gráfico No. 3}

\section{APLICACION DEL DIU DESPUES DE PARTO O ABORTO SEGUN CONTROL}

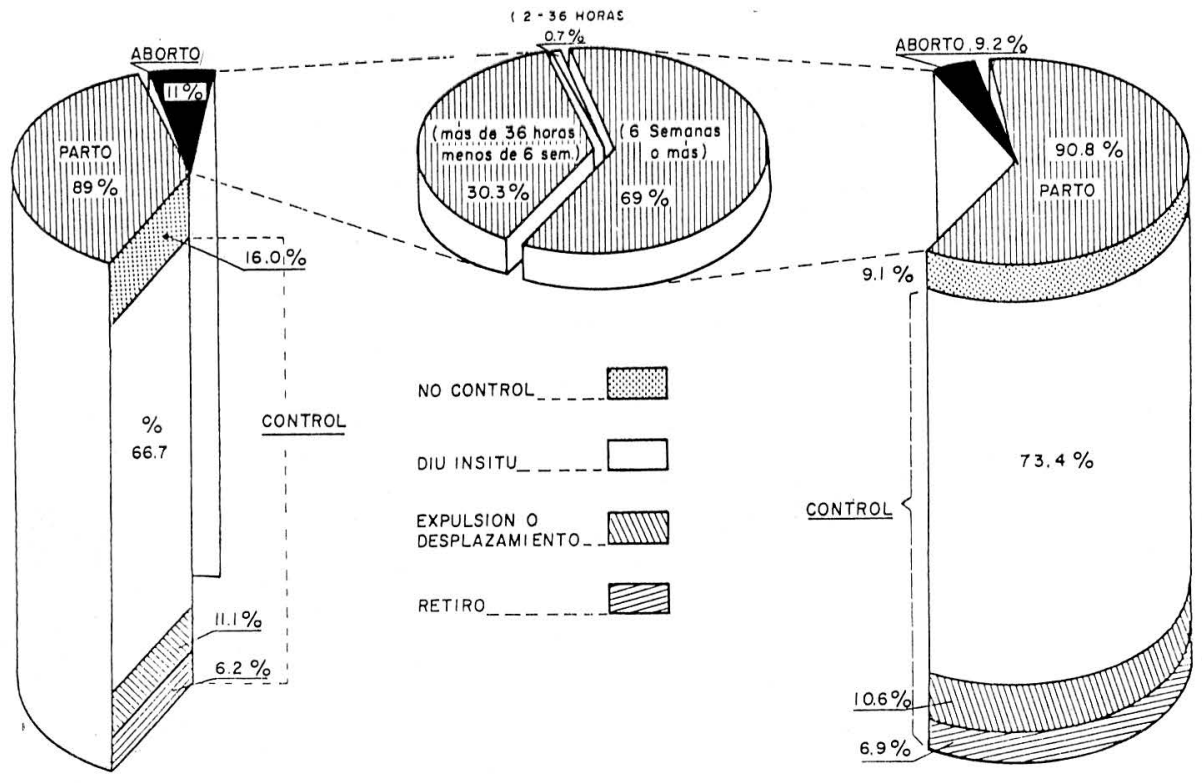

FUENTE: CCRP-PRIF. Inserción del DIU por personal paramédico. Hospital General de Medellin, 1.980 
tán relacionadas con las condiciones socio-económicas de la paciente 11 $(52.4 \%)$ que corresponden a 5 casos que no tienen quién cuide sus hijos $(23.8 \%), 3$ casos por problemas económicos $(14.3 \%)$ y 3 casos porque la paciente trabaja (14.3). (Tabla No. 18).

\section{Tiempo de aplicación del DIU y seguimiento}

Solamente a 2 pacientes se les aplicó el DIU entre las 2 a 36 horas después del parto, de éstas sólo 1 cumplió los 3 seguimientos, la otra paciente no cumplió ningún seguimiento (gráfico 30 ). De las 91 pacientes a las cuales se les aplicó el DIU entre las 36 horas y las 6 semanas de terminado el embarazo, 10 correspondieron a aborto $(11 \%)$ y 81 a parto $(89 \%)$; de las 10 pacientes de aborto: no se controló una paciente $(10 \%)$ y con control $9(90 \%)$.

De las 81 pacientes a las que se les aplicó después del parto $(89 \%), 13$ pacientes no asistieron a ningún control $(16 \%)$ y se controlaron $68(84 \%)$.

De 207 pacientes a las cuales se les aplicó el Dlú después de 6 semanas de terminado el embarazo, 188 fueron de parto $(90.8 \%)$ y 19 de aborto $(9.2 \%)$. De las 188 no volvieron $17(9.1 \%)$ y se controlaron $171(90.9 \%)$.

De las 19 pacientes de aborto, dos $(10.5 \%)$, no tuvieron ningún seguimiento, y se controlaron diecisiete $(89.5 \%)$.

Los hallazgos no presentan análisis comparativo entre pacientes usuarias del DIU después de aborto o después de

Gráfico No. 4

TOTAL DE PACIENTES SEGUN INTERRUPCION DEL METODO

\section{EN LOS TRES SEGUIMIENTOS}
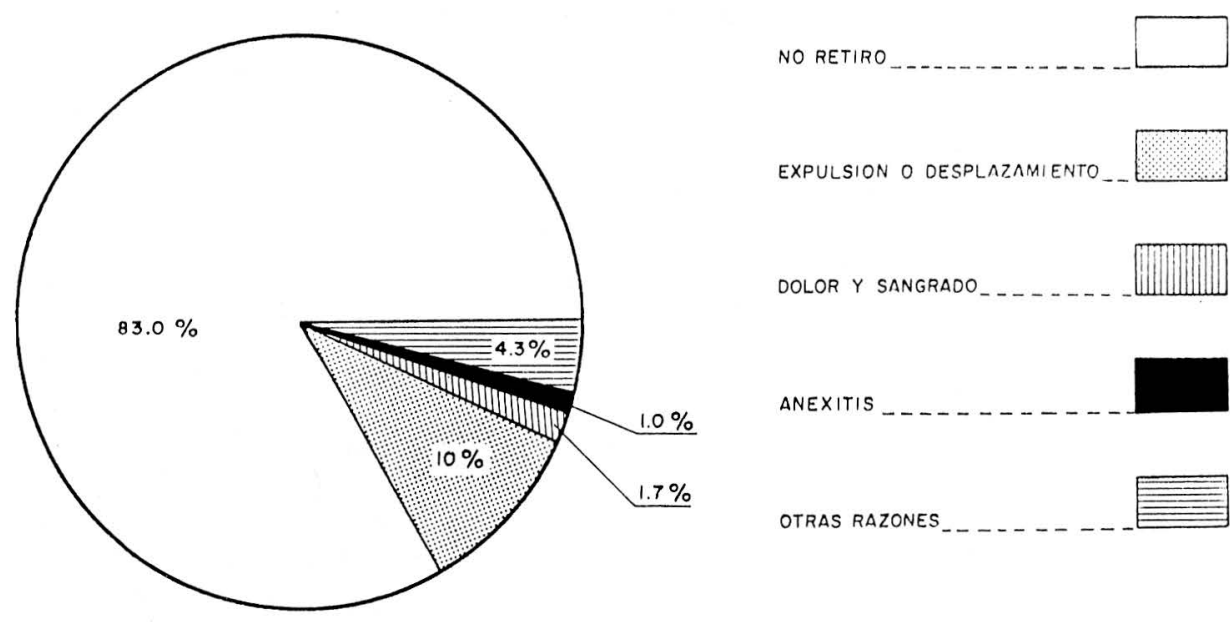

FUENTE: CCRP-PRIF. Inserción del DIU por personal poramédico. Hospital General de Medellin, 1.980 
Gráfico No. 5

\section{COMPARACION DE EXPULSION Y RETIRO DE DOS ESTUDIOS \\ EXTRANJEROS CON ESTE ESTUDIO}

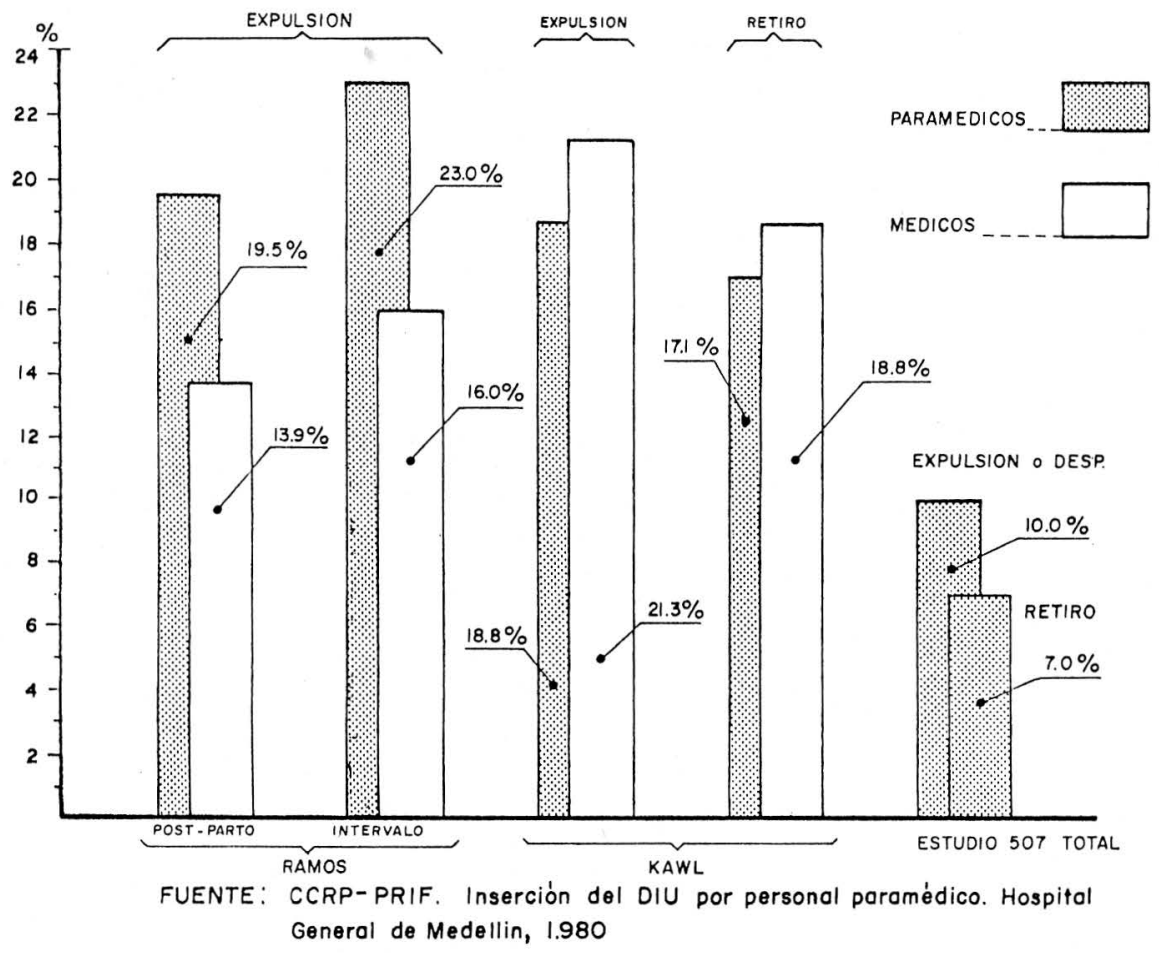

parto, en razón del bajo número de casos de aborto. Las ratas de expulsión o. retiro de los DIUs aplicados después del parto, no muestran diferencia significativa en el grupo de pacientes a los cuales se les aplicó el DIU entre las 36 horas y las 6 semanas de terminado el embarazo, con el grupo de pacientes a las cuales se aplicó después de 6 semanas de terminado el mismo.

Es importante señalar que si encontramos diferencia en el cumplimiento a
Ins controles en las pacientes de parto, ya que en el grupo de pacientes con parto reciente cumplieron el control el $84 \%$, mientras que en las pacientes con antecedente de parto de más de 6 semanas, el cumplimiento fue de $90.9 \%$. (Tabla No. 19 y gráfico 3).

24. Resui'tados totales de la interrupción del miótodo.

De los 300 DIUs apiicados sin diferenciar seguimientos o no, se presentaron 
51 interrupciones del método, distribuidas en 30 casos entre expulsiones y desplazamientos $(10 \%), 5$ retiros por dolor y sangrado $(1.7 \%), 3$ anexitis $(1.0 \%)$ y otras razones 15 casos $(4.3 \%)$. (Gráfico 4).

\section{Interrupción del método en los tres seguimientos}

Los resultados del primero y segundo seguimientos no muestran diferencia significativa en la interrupción del método. En el tercer seguimiento se encuentran pequeños incrementos en la expulsión y desplazamiento y la aparición de la anexitis como otra razón médica que se agrega, al dolor $y$ la hemorragia como razones para la extracción.

\section{Resultados totales de expulsión y desplazamiento sangrado y do- lor según tipo de dispositivo}

De las 30 expulsiones y desplazamientos, $22(73.3 \%)$ corresponden a Lippes C, y $8(26.7 \%)$ a Lippes D; lo que demuestra una diferencia significativa en la mayor probabilidad de expulsión y desplazamiento del Lippes $\mathrm{C}$ con relación al Lippes D. De los 5 retiros por dolor y sangrado 4 fueron Lippes $C$ $(80 \%)$ y una de Lippes D $(20 \%)$. Debi. do al reducido número de retiros, no se puede concluir nada definitivo comparativamente entre los dos tipos de dispositivos.

\section{Análisis comparativo con dos estudios extranjeros sobre e/ mis- mo tema}

Comparando los resultados totales de expulsión o desplazamiento y retiros, que fueron del $10 \%$ y $7 \%$ respectivamente, con las ratas encontradas por Ramos (13), que para el DIU aplicado en el post-parto por médicos fue de $13.9 \%$ y paramédicos $19.5 \%$ y los aplicados en intervalo por médicos fue de $16 \%$ y paramédicos $23 \%$; y Kawl (7) con ratas de expulsión para DIUs aplicados por médicos de $21.3 \%$, y paramédicos $18.8 \%$; retiros de $18.8 \%$ para DIUS aplicados por médicos y $17.1 \%$ para los paramédicos; podemos observar que nuestras ratas de expulsión y retiro son inferiores a las encontradas por estos dos investigadores. Gráfico 5.

\section{COMENTARIOS}

Como es común en la mayoría de los estudios realizados en nuestro medio, el cumplimiento de las pacientes a fin de llevar un adecuado seguimiento y control es muy pobre. En la actual investigación, el último control a los 6 meses de aplicado el DIU, sólo lo cumplieron 146 pacientes que constituyen el $48.7 \%$ del total. (Gráfico 1). Es importante destacar que en los 300 casos estudiados se encuentran 32 pacientes adolescentes $(10.7 \%)$, que es un porcentaje similar al encontrado en el estudio de atención del parto hospitalario realizado por la C.C. R.P. - P. R.I.F.,publicado en junio de 1979 y que fue de $8.19 \%(20)$.

Como era de esperarse en pacientes que solicitan un método anticonceptivo y que no son nulíparas, el resultado del último embarazo en su mayoría fue un hivo vivo $(89.3 \%)$. (Tabla No. 6).

El porcentaje alto de pacientes solicitantes que se encontraban lactando $(61.3 \%)$, nos demuestra la gran importancia del DIU como método de alta seguridad que no interfiere con la lactancia materna, lo que haría de él, un anticonceptivo ideal para ser aplicado a los 30 días post-parto, sin necesidad de esperar la primera menstruación después del parto, de terminar la lactancia, o de usar un método de barrera de baja efectividad.

A pesar de la supervisión, se les aplicó DIU a 4 pacientes, $(1.6 \%)$, con antece- 
dente de inflamación pélvica; esto podría indicar la necesidad de una mayor instrucción previa al grupo paramédico que se escoja para la aplicación de dispositivos intrauterinos.

Si se compara el antecedente de enfermedad venérea, que al ingreso era de $2 \%$; con la aparición de enfermedad venérea en el primer seguimiento que fue del $0.8 \%$, en el segundo seguimiento de $1.1 \%$, y en el tercer seguimiento de $0.7 \%$, podemos asumir que el uso del DIU no favorece al aumento de la enfermedad venérea en las pacientes estudiadas.

El análisis comparativo del tiempo de aplicación del DIU, con los hallazgos obtenidos en los tres seguimientos, entre Ios DIUs aplicados después del parto con los DIUs aplicados después del aborto, no admiten análisis comparativo por el bajo número de abortos.

El mejor cumplimiento a los controles del grupo de pacientes que tuvieron parto reciente (entre 36 horas y 6 semanas), puede explicarse por las exigencias de orden familiar; hallazgo común en nuestro medio, ya que el cumplimiento a la consulta de post-parto en la gran mayoría de las instituciones oficiales y privadas de Colombia es muy reducido.

Los resultados obtenidos en el presente estudio, demuestran que con un adiestramiento y supervisión adecuados, la aplicación del DIU puede delegarse en personal paramédico en nuestro medio, sin que esto modifique la efectividad del método o acentúe los efectos secundarios o complicaciones.

La aparición de la anexitis como complicación, se registro solamente al 60 . mes de la aplicación del DIU, lo que hace suponer que es una complicación tardía y que el riesgo de infección pélvica aumenta, a medida que aumenta el tiempo de uso del dispositivo.

\section{CONCLUSIONES}

1. La población usuaria fue joven, con un número importante de adolescentes como solicitantes del método.

2. No se encontró ninguna complicación grave al momento de la inserción, siendo la principal queja el dolor.

3. Sólo una paciente requirió tratamiento médico quirúrgico, por complicación al momento de la inserción.

4. No hubo ninguna muerte como complicación y la principal queia en los seguimientos fueron el sangrado anormal y el dolor.

5. El abandono del método fue bajo y la mayoría de las pacientes controladas. se encontraban satisfechas con el método.

6. Las ratas de expulsión y retiros en nuestro estudio, fueron inferiores a las encontradas por otros dos autores extranjeros en investigaciones similares.

7. Con adiestramiento y supervisión adecuados, la aplicación del DIU puede delegarse en personal paramédico, cuando las circunstancias as í lo exijan.

8. El riesgo de infección pélvica aumenta, a medida que aumenta el tiempo de uso del DIU.

\section{RESUMEN}

En el Hospital General de Medellín, se aplicaron 300 DIUs, tipo Lippes C y $D$, por personal paramédico que había recibido adiestramiento previo y con una adecuada supervisión.

La recolección de los datos se realizó en dos tipos de formularios precodificados, uno de admisión $y$ otro de seguimiento, el cual se extendió hasta los 6 meses después de aplicado el DIU. Los datos obtenidos fueron procesados por 
computador y posteriormente analizados. El análisis de los mismos demostró la bondad del procedimiento de delegar la aplicación del DIU a personal paramédico con adecuado adiestramiento y supervisión. No se encontró modificación con la efectividad, tolerancia, efectos secundarios o complicaciones comparándolo con estudios similares realizados en otros países.

\section{SUMMARY}

Previosuly trained paramedics inserted at the Medellin General Hospital 300 IUDs (Lippes Loops $C$ and D) supervised by medical personnel.

Data was collected in two types of precodified questionnaires. One filled in at registration other in a follow-up six months after insertion. Data collected was processed and analized. Analysis of data proved that trained paramedics under supervision can master the technical skills necessary for IUD insertion. Comparing the results of the study with

\section{BIBLIOGRAFIA}

1. AKIN A.; GRAY R.H.; RAMOS R.; "Training auxiliary nursemidwives to provide IUD services in Turkey and the Philippines". Stud fam plann 1980 May; 11 (5):178-87.

2. BIBB B.N. "The effectiveness of nonphysicians as providers of family planning services". Journal of obstetrics-ginecologic and neonatal nursing 1979 May-Jun; 8 (3): $137-43$.

3. CHOUDHURI A.S. "Role of paramedical personnel in the family planning pro gram in Pakistan". In: Zatuchni, G.I., ed. post partum family planning: a report on the international program. New York, N.Y., McGraw-hill, 1970 p. 185. 189.
La principal queja a la inserción fue el dolor y la principal causa de retiro del DIU a los seguimientos fue sangrado $y$ dolor.

La anexitis fue una complicación tardía que hace suponer que a mayor tiempo de uso del DIU, existe mayor riesgo de infección pélvica.

findings in studies carried out in other countries no substantial difference on effectivenes, rapport with clients, secondary effects or complications were found.

The main complaint was pain during insertion. The principal cause for removals was bleeding and pain.

Anexitis resulted to be a late complication wich permits to assume that PID is more likely to occur with longer IUD use.

4. FEPAFEN. "Enseñanza de la planifica ción familiar en las facultades (escuelas) de medicina. Plan básico. Bogotá, Colombia, junio 1974.

5. HARTFIELD V.J. "The role of parame dical personnel in family planning programmes with particular reference to intrauterine devices". West african medical journal 17: 225-226. December 1968 .

6. JAFAREY S.A. HARDEE; SATTERTHWAITE A,P. "Use of medical paramedical personnel and traditional midwives, in the Pakistan family planning program': Demography 5 (2): 666-678. 1968.

7. KAUL S.J. "A comparison of field perfomance of medical vs. paramedical per- 
sonnel in the IUD programme in mymensingh and lahore". Pakistan journal of family planning 3: 75-93. July 1969.

8. KLEINMAN R.L. "Family planning for midwives and nurses". London Interna tional planned parenthood federation, 1971.

9. MANISOFF M,T. "Planeación familiar". Guía para enfermeras. Editorial Intera mericana México. D.F. Diciembre 1975.

10. OCHOA LUIS HERNANDO. "Seminario sobre estrategias y ubicación de la planificación familiar en Colombia". Bogotá, marzo 27 de 1981 CCRP-PRIF.

11. PECK P.R, "The role of paramedical personnel in family planning': In Polgar, S., ed. Culture and Population: A collection of current studies. Carolina Popula tion Center University of North Carolina at chapel hill, p. 167-175.

12. PHILLIPS-GAY M. "The role of paramedical personnel" In: proceedings of the eighth international conference of I.P.P. F., Santiago, Chile, 204-206. Abril 9-15, a 1967.

13. RAMOS R.: APELO R.A. "A program of IUD insertions by paraprofessionals and physicians in the Philippines". Int. J. Gynaecol obstet. 1978-1979; $16(4)$ : 321-3.

14. REAME N.E. "Role of paramedical personnel in IUD and family planning". Contraceptive delivery sistems. 1980 Jul. ; 1 (3); $277-8$.

15. ROSENFIELD A.G. "Nursing and auxi liary personnel: a training guide for family planning programs". Advances in planned parenthood 11 (4): $185-190$ 1976.

16. ROSENFIELD A.G. "The IUD in family planning programs: programatic issues". In: Hefnawi, F. and Segal, S.J. eds. proceedings of the third international conference on intrauterine contraception,
Cairo, Egypt, December 12-14, 1974, Amsterdam, North-Holland, 1975 p. 34 45.

17. ROSENFIELD A.G. "Laws relating to paraprofessional role in contraception". Background paper prepared for the symposium on law and population, Tunis, Tunis, june 17-21, 1974. p. 19.

18. SATTERTHWAITE A.P. "Training and perfomance of paramedical personnel in the Pakistan family planning program me'". In: fourth Asian congress of Obstetrics and Gynaecology scientific subcommitee eds. The congress volume of papers and abstracts, Singapore, November, 15-24, 1968. (Singapore, Eurasia Press; 1969). p. 99.

19. SINGH R.P.; TALWAR P.P. "Lippe's loop insertions by doctors and paramedical staff: A comparative Study". Pop. cen. Newsletter 5 (3): 1-7 June-Jule. 1979.

20. URIZA G. "Embarazo en adolescentes". Conferencia presentada en el curso de Ginecología Infanto Juvenil. Cúcuta, $\propto$ tubre 9-11de 982

21. ZEIGHAMI E.; ZEIGHAMI B.; EFTEKHARI A.E, ; KHOSHNEVIS P. "Effectiveness of the Iranian auxiliary midwife in IUD insertion". Studies in family planning 7 (9): 261-263, september 1976.

\section{AGRADECIMIENTO}

El área Biomédica de la Corporación Centro Regional de Población y los autores del presente estudio, agradecen la efectiva colaboración de las Directivas y el Personal del Hospital General de Medellín y en forma muy especial a la doctora Libia Restrepo de Ramírez Jefe del departamento de Ginecología y Obstetricia. Sin esta ayuda no hubiese sido posible realizar la presente investigación. 